\title{
USO DE ATMOSFERA CONTROLADA PARA CONSERVAÇÃO PÓS-COLHEITA DO MAMÃO 'GOLDEN' 1
}

\author{
MARCOS JOSÉ DE OLIVEIRA FONSECA², SÉRGIO AGOSTINHO CENCI ${ }^{3}$, NILTON ROCHA LEAL ${ }^{4}$, NEIDE BOTREL ${ }^{3}$
}

RESUMO - Frutos de mamoeiro 'Golden' colhidos no estádio 3 de maturação foram armazenados sob controle de atmosfera $\left(3 \% \mathrm{O}_{2}\right.$ e $3 \% \mathrm{CO}_{2}, 3 \% \mathrm{O}_{2}$ e $6 \% \mathrm{CO}_{2}$ ou $8 \% \mathrm{CO}_{2}, 5 \% \mathrm{O}_{2}$ e $8 \% \mathrm{CO}_{2}$ ) e sob atmosfera ambiente ( $70 \%$ ou $90 \%$ U.R.), por 20 ou 25 dias, seguidos de mais 5 dias em atmosfera ambiente, a $10^{\circ} \mathrm{C}$. Após 4 dias a $22^{\circ} \mathrm{C}$ não se verificaram muitas diferenças entre tratamentos, porém o maior teor de sólidos solúveis em frutos armazenados sob $3 \% \mathrm{O}_{2} / 3 \% \mathrm{CO}_{2}$, e os maiores índices de maturidade nos frutos armazenados sob $3 \% \mathrm{O}_{2} / 6 \% \mathrm{CO}_{2}$, por 20 dias, sugerem que novos estudos possam ser realizados adotando-se tais concentrações.

Termos para indexação: Carica papaya L., atmosfera controlada, pós-colheita, exportação.

\section{THE USE OF CONTROLLED ATMOSPHERE FOR POSTHARVEST CONSERVATION OF ‘GOLDEN' PAPAYA}

Abstract - 'Golden' papaya was harvested in stage 3 of a maturation scale, storaged in four controlled atmospheres $\left(3 \% \mathrm{O}_{2}\right.$ and $3 \% \mathrm{CO}_{2}, 3 \% \mathrm{O}_{2}$ and $6 \% \mathrm{CO}_{2}$ or $8 \% \mathrm{CO}_{2}$, and $5 \% \mathrm{O}_{2}$ and $8 \% \mathrm{CO}_{2}$ ) and two ambient atmosphere conditions ( $70 \%$ R.H. and $90 \%$ R.H.), for 20 or 25 days, plus 5 days of ambient atmosphere at $10^{\circ} \mathrm{C}$. After for 4 days, at $22^{\circ} \mathrm{C}$, it was not observed differences between treatments, but the greatest total soluble solids, in fruits storaged under $3 \% \mathrm{O}_{2} / 6 \% \mathrm{CO}_{2}$, and the greatest ratio of fruits storage under $3 \% \mathrm{O}_{2} / 6 \% \mathrm{CO}_{2}$, for 20 days, which suggests that new researches may be conducted with these controlled atmospheres.

Index Terms: Carica papaya L., controlled atmosphere, post-harvest, exportation.

As exportações brasileiras de mamão do grupo Solo geraram 18,5 milhões de dólares de divisas para o Brasil em 2001, contribuindo para o superávit do agronegócio nacional. Entretanto, as perdas são entrave para a exportação via marítima, que reduziria o custo do frete, aumentando a competitividade no mercado mundial. O controle da atmosfera pode ser implantada, a partir do momento em que se verifique possibilidade de extensão do período de conservação, sem o comprometimento da qualidade sensorial. Não existem muitos estudos sobre o uso de atmosfera controlada para o mamão. Kader (1999) recomenda, para os mamões do grupo Solo, atmosferas de 3 a $5 \%$ de $\mathrm{O}_{2}$ e de 5 a $8 \%$ de $\mathrm{CO}_{2}$. O objetivo deste trabalho foi o de estabelecer uma atmosfera controlada na preservação da qualidade pós-colheita do mamão 'Golden', simulando-se condições de transporte e comercialização no mercado europeu.

Os frutos de mamoeiro 'Sunrise Solo' foram colhidos no estádio 3 (25 a 40\% da área superficial amarela) da escala de maturação desenvolvida por Balbino (1997), em pomar comercial localizado em Linhares-ES. Os frutos foram submetidos à hidrotermia e imersão em calda fungicida, classificados (10 frutos por caixa), embalados em caixas de papelão tipo exportação e transportados refrigerados $\left(10^{\circ} \mathrm{C}\right)$ para a Embrapa Agroindústria de Alimentos, Rio de Janeiro-RJ, onde foram submetidos à temperatura de $10^{\circ} \mathrm{C}, 85-95 \%$ de umidade relativa, e a cinco atmosferas (ambiente; $3 \% \mathrm{O}_{2} / 3 \% \mathrm{CO}_{2} ; 3 \% \mathrm{O}_{2} / 6 \% \mathrm{CO}_{2} ; 3 \% \mathrm{O}_{2} / 8 \% \mathrm{CO}_{2}$ e $5 \% \mathrm{O}_{2} / 8 \% \mathrm{CO}_{2}$ ). Foi estabelecido controle em atmosfera ambiente e a umidade relativa de $70 \%$. Os frutos foram retirados destas condições, após 20 ou 25 dias, período mínimo e máximo de transporte marítimo. Em seguida, foram submetidos a $22^{\circ} \mathrm{C}$, por 12 horas, período correspondente ao deslocamento para o mercado atacadista. Após isso, os frutos foram mantidos por cinco dias a $10^{\circ} \mathrm{C}$, simulando a comercialização atacadista no mercado europeu. Os frutos foram avaliados nas condições de comercialização no varejo $\left(25^{\circ} \mathrm{C}\right)$, aos 0 e 4 dias, para ambos os tempos de transporte, quanto às seguintes características: desenvolvimento de cor de polpa, em relação à escala desenvolvida por Balbino (1997); perda de massa fresca, em relação à massa inicial; firmeza de polpa, após a remoção da casca, em quatro pontos eqüidistantes na região mediana, medindo-se a resistência à penetração da ponteira de penetrômetro tipo EFFEGGI (5/16"), expressando-se os resultados em $\mathrm{kg} \cdot \mathrm{m}^{-2} \cdot 10^{-4}$ (Fonseca, 2002); sólidos solúveis totais (SST), com o uso de refratômetro (ATAGO N1) e valor expresso em ${ }^{\circ}$ Brix (Adolfo Lutz, 1985) e acidez titulável (AT) em percentagem de ácido cítrico, pela titulação de alíquota do suco com solução $0,1 \mathrm{~N}$ de $\mathrm{NaOH}$, em presença de fenolftaleína (pH final: 8,0) (Adolfo Lutz, 1985).

Os tratamentos foram dispostos em parcelas subdivididas, em que atmosferas de armazenamento constituíram as parcelas e as datas de avaliações constituíram as subparcelas, em delineamento em blocos casualisados, com três repetições. Os dados foram interpretados por análise de variância e teste de Tukey, a 5\% de probabilidade.

Para o primeiro dia de comercialização varejista (viagem marítima mais curta), a maior retenção da firmeza foi verificada em mamão 'Golden', armazenado sob $5 \% \mathrm{O}_{2}$ e $8 \% \mathrm{CO}_{2}$ e $3 \% \mathrm{O}_{2}$ e $6 \% \mathrm{CO}_{2}$, embora a firmeza destes últimos não diferisse das observadas nos frutos armazenados sob atmosfera ambiente, independentemente da umidade relativa (Tabela 1). Após 4 dias, não mais se verificaram diferenças entre os frutos. Após 25 dias, verificou-se maior firmeza nos frutos armazenados sob atmosfera ambiente ( $90 \%$ U.R.) e nas atmosferas contendo $3 \% \mathrm{O}_{2}$ e $8 \% \mathrm{CO}_{2}$ ou $3 \% \mathrm{CO}_{2}$. Da mesma forma que nos frutos submetidos à simulação de 20 dias, após 4 dias não se verificou diferença na firmeza de polpa dos frutos.

A perda de massa fresca do mamão 'Golden' não diferiu entre os tratamentos na simulação de armazenamento por 20 dias (Tabela 2). Com exceção dos frutos armazenados sob $5 \% \mathrm{O}_{2}$ e $8 \% \mathrm{CO}_{2}$, a perda de massa foi inferior a $5 \%$. No armazenamento por 25 dias, também não se verificou diferença na perda de massa dos frutos. Somente após quatro dias de simulação pode-se observar menor perda de massa nos frutos armazenados sob atmosfera ambiente e $90 \%$ de umidade relativa. A perda de massa dos frutos não diferiu entre as atmosferas controladas, nesta data de avaliação. A menor firmeza de polpa e a maior perda de massa fresca dos frutos armazenados na atmosfera contendo $5 \%$ de $\mathrm{O}_{2}$, após 20 dias, podem estar indicando maior respiração e amadurecimento mais acelerado, em relação aos frutos armazenados sob 3\% de $\mathrm{O}_{2}$.

Os sólidos solúveis totais do mamão 'Golden' não diferiram entre os tratamentos, no período mais longo de armazenamento (25 dias), nas duas datas avaliadas ( 0 e 4 dias) (Tabela 3). Possivelmente, os frutos já teriam completado seu amadurecimento. Por outro lado, verificou-se maiores teores de sólidos solúveis totais nos frutos armazenados sob atmosfera contendo $3 \% \mathrm{O}_{2}$ e $8 \% \mathrm{CO}_{2}, 3 \% \mathrm{CO}_{2}$ ou $6 \% \mathrm{CO}_{2}$ e atmosfera am-

${ }^{1}$ (Trabalho 155/2002). Recebido: 20/09/2002. Aceito para publicação: 11/09/2003.

2 D.Sc. Pesquisador - EMBRAPA Milho e Sorgo - Rod. MG 424 km 65, C.P. 151- Sete Lagoas/MG - CEP 35701-970 - (31) $3779-1035$. mfonseca@cnpms.embrapa.br

${ }^{3}$ D.Sc. pesquisador - EMBRAPA Agroindústria de Alimentos

${ }^{4} \mathrm{Ph}$.D. Professor Titular - Universidade Estadual do Norte Fluminense 
TABELA 1 - Valores médios de firmeza de polpa de mamão 'Golden', armazenado sob atmosfera controlada, contendo 3\% $\mathrm{O}_{2}$ e $8 \% \mathrm{CO}_{2}, 5 \% \mathrm{O}_{2}$ e $8 \% \mathrm{CO}_{2}$, $3 \% \mathrm{O}_{2}$ e $3 \% \mathrm{CO}_{2}$ ou $3 \% \mathrm{O}_{2}$ e $6 \% \mathrm{CO}_{2}$ e sob atmosfera ambiente com $70 \%$ U.R. ou com $90 \%$ U.R. nas respectivas datas de avaliação (Rio de Janeiro, 2000)

\begin{tabular}{lllcc}
\hline \multicolumn{1}{c}{ Atmosfera de Conservação } & \multicolumn{3}{c}{ Firmeza de Polpa $\left[\mathrm{kg}\left(\mathrm{m}^{2} .10^{-4}\right)^{-1}\right]$} \\
\cline { 2 - 5 } & $20+5+0$ dias & $20+5+4$ dias & $25+5+0$ dias & $25+5+5$ dias \\
\hline $3 \% \mathrm{O}_{2}$ e $8 \% \mathrm{CO}_{2}$ & $4,1 \mathrm{c}$ & $4,3 \mathrm{a}$ & $8,7 \mathrm{abc}$ & $1,8 \mathrm{a}$ \\
$5 \% \mathrm{O}_{2}$ e $8 \% \mathrm{CO}_{2}$ & $9,0 \mathrm{a}$ & $4,3 \mathrm{a}$ & $6,0 \mathrm{~cd}$ & $2,4 \mathrm{a}$ \\
$3 \% \mathrm{O}_{2}$ e $3 \% \mathrm{CO}_{2}$ & $4,9 \mathrm{c}$ & $4,1 \mathrm{a}$ & $10,9 \mathrm{ab}$ & $1,2 \mathrm{a}$ \\
$3 \% \mathrm{O}_{2}$ e $6 \% \mathrm{CO}_{2}$ & $8,1 \mathrm{ab}$ & $7,2 \mathrm{a}$ & $8,2 \mathrm{bc}$ & $1,2 \mathrm{a}$ \\
Atmosfera ambiente com $70 \%$ U.R. & $5,5 \mathrm{bc}$ & $6,0 \mathrm{a}$ & 5,0 & $\mathrm{~d}$ \\
Atmosfera ambiente com $90 \%$ U.R. & $5,0 \mathrm{bc}$ & $5,8 \mathrm{a}$ & $11,4 \mathrm{a}$ & $1,2 \mathrm{a}$ \\
\hline
\end{tabular}

Médias seguidas de pelo menos uma mesma letra na coluna, não diferem entre si, pelo teste de Tukey, a 5\% de probabilidade.

TABELA2 - Valores médios de percentual de perda de massa de mamão 'Golden', armazenado sob atmosfera controlada, contendo 3\% $\mathrm{O}_{2}$ e $8 \% \mathrm{CO}_{2}$, $5 \% \mathrm{O}_{2}$ e $8 \% \mathrm{CO}_{2}, 3 \% \mathrm{O}_{2}$ e $3 \% \mathrm{CO}_{2}$ ou $3 \% \mathrm{O}_{2}$ e $6 \% \mathrm{CO}_{2}$ e sob atmosfera ambiente com $70 \%$ U.R. ou com $90 \%$ U.R. nas respectivas datas de avaliação (Rio de Janeiro, 2000)

\begin{tabular}{lcccc}
\hline \multicolumn{1}{c}{ Atmosfera de Conservação } & \multicolumn{3}{c}{ Percentual de Perda de Massa } \\
\cline { 2 - 5 } & $20+5+0$ dias & $20+5+4$ dias & $25+5+0$ dias & $25+5+5$ dias \\
\hline $3 \% \mathrm{O}_{2}$ e $8 \% \mathrm{CO}_{2}$ & $2,0 \mathrm{a}$ & $4,3 \mathrm{a}$ & $3,5 \mathrm{a}$ & $8,4 \mathrm{ab}$ \\
$5 \% \mathrm{O}_{2}$ e $8 \% \mathrm{CO}_{2}$ & $2,5 \mathrm{a}$ & $5,4 \mathrm{a}$ & $3,3 \mathrm{a}$ & $10,0 \mathrm{a}$ \\
$3 \% \mathrm{O}_{2}$ e $3 \% \mathrm{CO}_{2}$ & $2,0 \mathrm{a}$ & $4,5 \mathrm{a}$ & $2,7 \mathrm{a}$ & $9,4 \mathrm{a}$ \\
$3 \% \mathrm{O}_{2}$ e $6 \% \mathrm{CO}_{2}$ & $1,8 \mathrm{a}$ & $4,8 \mathrm{a}$ & $2,9 \mathrm{a}$ & $2,1 \mathrm{a}$ \\
$\mathrm{Atmosfera} \mathrm{ambiente} \mathrm{com} 70 \%$ U.R. & $1,8 \mathrm{a}$ & $4,0 \mathrm{a}$ & $1,5 \mathrm{a}$ & $5,8 \mathrm{bc}$ \\
Atmosfera ambiente com $90 \%$ U.R. & $2,3 \mathrm{a}$ & $4,4 \mathrm{a}$ & $\mathrm{c}$ & 3,9 \\
\hline
\end{tabular}

Médias seguidas de pelo menos uma mesma letra na coluna, não diferem entre si, pelo teste de Tukey, a 5\% de probabilidade.

TABELA3 - Valores médios de sólidos solúveis totais de mamão 'Golden', armazenado sob atmosfera controlada, contendo 3\% $\mathrm{O}_{2}$ e $8 \% \mathrm{CO}_{2}, 5 \% \mathrm{O}_{2}$ e $8 \% \mathrm{CO}_{2}, 3 \% \mathrm{O}_{2}$ e $3 \% \mathrm{CO}_{2}$ ou $3 \% \mathrm{O}_{2}$ e $6 \% \mathrm{CO}_{2}$ e sob atmosfera ambiente com $70 \%$ U.R. ou com $90 \%$ U.R. nas respectivas datas de avaliação (Rio de Janeiro, 2000)

\begin{tabular}{llcll}
\hline \multirow{2}{*}{ Atmosfera de Conservação } & \multicolumn{4}{c}{ Sólidos Solúveis Totais $\left({ }^{\circ}\right.$ Brix $)$} \\
\cline { 2 - 5 } & $20+5+0$ dias & $20+5+4$ dias & $25+5+0$ dias & $25+5+5$ dias \\
\hline $3 \% \mathrm{O}_{2}$ e $8 \% \mathrm{CO}_{2}$ & $12,0 \mathrm{ab}$ & $11,7 \mathrm{bc}$ & $11,7 \mathrm{a}$ & $11,4 \mathrm{a}$ \\
$5 \% \mathrm{O}_{2}$ e $8 \% \mathrm{CO}_{2}$ & $11,5 \mathrm{~b}$ & $11,8 \mathrm{bc}$ & $11,7 \mathrm{a}$ & $11,1 \mathrm{a}$ \\
$3 \% \mathrm{O}_{2}$ e $3 \% \mathrm{CO}_{2}$ & $13,1 \mathrm{a}$ & $13,7 \mathrm{a}$ & $11,5 \mathrm{a}$ & $1,9 \mathrm{a}$ \\
$3 \% \mathrm{O}_{2}$ e $6 \% \mathrm{CO}_{2}$ & $12,1 \mathrm{ab}$ & $13,2 \mathrm{ab}$ & $12,1 \mathrm{a}$ & $11,7 \mathrm{a}$ \\
$\mathrm{Atmosfera} \mathrm{ambiente,} 70 \%$ U.R. & $11,3 \mathrm{~b}$ & $11,1 \mathrm{c}$ & $11,6 \mathrm{a}$ & $10,5 \mathrm{a}$ \\
Atmosfera ambiente, 90\% U.R. & $11,9 \mathrm{ab}$ & $11,5 \mathrm{bc}$ & $10,8 \mathrm{a}$ & $11,8 \mathrm{a}$ \\
\hline
\end{tabular}

Médias seguidas de pelo menos uma mesma letra na coluna, não diferem entre si, pelo teste de Tukey, a $5 \%$ de probabilidade.

biente (90\% U.R.), no primeiro dia de avaliação, na simulação de 20 dias. Os teores mantiveram-se elevados somente nos frutos armazenados sob $3 \% \mathrm{O}_{2}$ e $3 \% \mathrm{CO}_{2}$ ou $6 \% \mathrm{CO}_{2}$. Na Tabela 4 , não se verificam diferenças na acidez titulável, podendo-se inferir que não houve redução do metabolismo dos frutos.

A razão entre sólidos solúveis totais e acidez titulável é uma característica que reflete a qualidade sensorial de frutos, sendo conhecida também como índice de maturidade (Agustí, 2000). Na simulação de período mais curto de armazenamento, foram observados maiores valores desta razão nos frutos conservados sob atmosfera controlada contendo $3 \% \mathrm{O}_{2}$ e $3 \% \mathrm{CO}_{2}$ ou $6 \% \mathrm{CO}_{2}$, e ambiente (70\% U.R.) (Tabela 5). Esta superioridade somente se manteve na atmosfera contendo $3 \% \mathrm{O}_{2}$ e $6 \% \mathrm{CO}_{2}$ e na atmosfera ambiente com $70 \%$ de umidade relativa, após 4 dias. Os maiores índices de maturidade encontrados nestas duas datas suportam que o armazenamento, por 20 dias, em atmosfera contendo $3 \% \mathrm{O}_{2}$ e $6 \% \mathrm{CO}_{2}$, proporcionou maior qualidade sensorial ao mamão 'Golden'. A diferenciação entre tratamentos para este índice deveu-se principalmente ao teor de sólidos solúveis, sobretudo quanto às atmosferas $3 \%$ de $\mathrm{O}_{2}$ e $3 \%$ ou $6 \%$ de $\mathrm{CO}_{2}$. Verificam-se poucas diferenças nesta característica, para os frutos armazenados por 25 dias sob controle ou não da atmosfera (Tabela 5).

A não diferenciação da coloração de casca do mamão 'Golden' pode ser devida a sua cor mais clara em relação a outras cultivares (Tabela 5). Marin \& Gomes (2000) descrevem o cultivar como acentuadamente aclorofilado. Fonseca (2002) comparando seus teores de clorofila $a, b$ e total, com os do mamão 'Sunrise Solo', constatou que o mamão
'Golden' possui teores significativamente menores nos 6 primeiros estádios de maturação, em escala de 1 a 7.

A pouca diferenciação entre os tratamentos pode ser devida à temperatura adotada para os estudos (consagrada entre os exportadores), suficiente para reduzir o metabolismo em tamanha magnitude, que o processo respiratório tenha se mantido em nível de manutenção (Salisbury \& Ross, 1992). A menor disponibilidade de oxigênio pode ter causado anaerobiose, promovendo acúmulo de etanol que pode estimular biossíntese de etileno, desencadeando a maturação dos frutos (Burdon et al., 1996). A produção de etanol, apesar de não ter sido medida, pode ter ocorrido, uma vez que ao se provar a polpa dos frutos, armazenados sob atmosfera controlada, pode ser verificada a ocorrência de sabor e aroma desagradáveis.

Apesar das poucas evidências de incremento ou manutenção de qualidade sob controle da atmosfera, o maior teor de sólidos solúveis do mamão 'Golden' armazenado sob 3\% $\mathrm{O}_{2}$ e 3\% $\mathrm{CO}_{2}$, e os maiores índices de maturidade sob $3 \% \mathrm{O}_{2}$ e $6 \% \mathrm{CO}_{2}$ por 20 dias, qualificam tais atmosferas para estudos mais avançados. Porém, há a necessidade de que outras características sejam medidas como, por exemplo, atividade respiratória, produção de etileno e de etanol. A inexistência de diferenças entre os mamões 'Golden', armazenados por 25 dias, indica que este período de tempo é longo para a eficiência da tecnologia.

Os autores manifestam seus sinceros agradecimentos a White Martins pelo fornecimento das misturas gasosas, à Empresa Gaia, pelos frutos e à UENF/FENORTE pela bolsa de estudos. 
TABELA4 - Valores médios de acidez titulável de mamão 'Golden', armazenado sob atmosfera controlada, contendo 3\% $\mathrm{O}_{2}$ e $8 \% \mathrm{CO}_{2}, 5 \% \mathrm{O}_{2}$ e $8 \% \mathrm{CO}_{2}$, $3 \% \mathrm{O}_{2}$ e $3 \% \mathrm{CO}_{2}$ ou $3 \% \mathrm{O}_{2}$ e $6 \% \mathrm{CO}_{2}$ e sob atmosfera ambiente com $70 \%$ U.R. ou com $90 \%$ U.R. nas respectivas datas de avaliação (Rio de Janeiro, 2000)

\begin{tabular}{lccc}
\hline \multicolumn{1}{c}{ Atmosfera de Conservação } & \multicolumn{2}{c}{ Acidez Titulável [g ácido cítrico(100g de polpa) $^{-1}$ ] } \\
\cline { 2 - 4 } & $20+5+0$ dias & $20+5+4$ dias & $25+5+0$ dias \\
\hline $3 \% \mathrm{O}_{2}$ e $8 \% \mathrm{CO}_{2}$ & $0,161 \mathrm{a}$ & $0,165 \mathrm{a}$ & $0,151 \mathrm{a}$ \\
$5 \% \mathrm{O}_{2}$ e $8 \% \mathrm{CO}_{2}$ & $0,166 \mathrm{a}$ & $0,148 \mathrm{a}$ & $0,117 \mathrm{a}$ \\
$3 \% \mathrm{O}_{2}$ e $3 \% \mathrm{CO}_{2}$ & $0,129 \mathrm{a}$ & $0,144 \mathrm{a}$ & $0,164 \mathrm{a}$ \\
$3 \% \mathrm{O}_{2}$ e $6 \% \mathrm{CO}_{2}$ & $0,115 \mathrm{a}$ & $0,125 \mathrm{a}$ & $0,134 \mathrm{a}$ \\
$\mathrm{Atmosfera} \mathrm{ambiente} \mathrm{com} 70 \%$ U.R. & $0,125 \mathrm{a}$ & $0,137 \mathrm{a}$ & $0,189 \mathrm{a}$ \\
Atmosfera ambiente com $90 \%$ U.R. & $0,159 \mathrm{a}$ & $0,105 \mathrm{a}$ & $0,162 \mathrm{a}$ \\
\hline
\end{tabular}

Médias seguidas de pelo menos uma mesma letra na coluna, não diferem entre si, pelo teste de Tukey, a 5\% de probabilidade.

TABELA 5 - Valores médios da razão entre sólidos solúveis totais e acidez titulável de mamão 'Golden', armazenado sob atmosfera controlada, contendo $3 \% \mathrm{O}_{2}$ e $8 \% \mathrm{CO}_{2}, 5 \% \mathrm{O}_{2}$ e $8 \% \mathrm{CO}_{2}, 3 \% \mathrm{O}_{2}$ e $3 \% \mathrm{CO}_{2}$ ou $3 \% \mathrm{O}_{2}$ e $6 \% \mathrm{CO}_{2}$ e sob atmosfera ambiente com $70 \%$ U.R. ou com $90 \%$ U.R. nas respectivas datas de avaliação (Rio de Janeiro, 2000)

\begin{tabular}{lcccc}
\hline \multicolumn{1}{r}{ Atmosfera de Conservação } & \multicolumn{4}{c}{ Razão entre sólidos solúveis totais e acidez titulável } \\
\cline { 2 - 5 } & $20+5+0$ dias & $20+5+4$ dias & $25+5+0$ dias & $25+5+5$ dias \\
\hline $3 \% \mathrm{O}_{2}$ e $8 \% \mathrm{CO}_{2}$ & $75,5 \mathrm{bc}$ & $71,1 \mathrm{~b}$ & $78,6 \mathrm{ab}$ & $77,2 \mathrm{ab}$ \\
$5 \% \mathrm{O}_{2}$ e $8 \% \mathrm{CO}_{2}$ & $69,7 \mathrm{c}$ & $82,5 \mathrm{ab}$ & $100,4 \mathrm{a}$ & $77,5 \mathrm{ab}$ \\
$3 \% \mathrm{O}_{2}$ e $3 \% \mathrm{CO}_{2}$ & $103,6 \mathrm{ab}$ & $76,2 \mathrm{~b}$ & $72,1 \mathrm{~b}$ & $74,9 \mathrm{ab}$ \\
$3 \% \mathrm{O}_{2}$ e $6 \% \mathrm{CO}_{2}$ & $104,9 \mathrm{a}$ & $106,0 \mathrm{a}$ & $90,0 \mathrm{ab}$ & $67,8 \mathrm{~b}$ \\
Atmosfera ambiente com $70 \%$ U.R. & $90,1 \mathrm{abc}$ & $81,1 \mathrm{ab}$ & $79,4 \mathrm{ab}$ & $101,1 \mathrm{a}$ \\
Atmosfera ambiente com $90 \%$ U.R. & $75,8 \mathrm{bc}$ & $85,5 \mathrm{ab}$ & $68,1 \mathrm{~b}$ & $74,0 \mathrm{ab}$ \\
\hline
\end{tabular}

Médias seguidas de pelo menos uma mesma letra na coluna, não diferem entre si, pelo teste de Tukey, a $5 \%$ de probabilidade.

\section{REFERÊNCIAS BIBLIOGRÁFICAS}

ADOLFO LUTZ. Normas analíticas do Instituto Adolfo Lutz.: Métodos

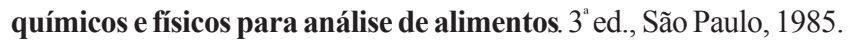
v. 1.

AGUSTÍ, M. Crecimiento y maduración del fruto. In: AZCÓN-BIETO, J.; TALÓN, M. Fundamentos de Fisiología Vegetal. Barcelona: Edicions Universitat de Barcelona, 2000. p.419-433.

BALBINO, J.M.S. Efeitos de hidrotermia, refrigeração e ethephon na qualidade pós-colheita do mamão (Carica papaya L.). 1997. 104 f. Tese (Doutorado em Fisiologia Vegetal) - Universidade Federal de Viçosa, Viçosa,1997.

BURDON, J.; DORI, S.; MARINANSKY, R.; PESIS, E. Acetaldehyde inhibition of ethylene in mango fruit. Postharvest Biology and Tech- nology, v.8, p.153-161, 1996.

FONSECA, M.J.O. Conservação pós-colheita de mamão (Carica papaya L.): análise das cultivares Sunrise Solo e Golden, sob controle de temperatura e da atmosfera, 2002. 177f. Tese (Doutorado em Produção Vegetal) Universidade Estadual do Norte Fluminense, Campos dos Goytacazes, 2002

KADER,A.A. Papaya: recomendations for maintaining postharvest quality. Davis: University of California, 1999. 4p.

MARIN, S.L.D., GOMES, J.A. Cultura do mamão. In: Instituto FRUTAL. 7 "Semana Internacional de Fruticultura e Agroindústria. Fortaleza: Instituto FRUTAL, 2000. 50p.

SALISBURY, FB., ROSS, C.W.Plant Physiology. California: Wadsworth Publishing Company, 1992. 682p. 